\title{
Management of the First Patellar Dislocation: A Narrative Review
}

\author{
Erica Bulgheroni ${ }^{1}$ Michele Vasso ${ }^{2}$ Michele Losco ${ }^{3}$ \\ Luciano Bertoldi ${ }^{1}$ Alfredo Schiavone Panni ${ }^{2}$ \\ ${ }^{1}$ Department of Orthopaedic and Trauma Surgery, Santa Chiara \\ Hospital, Trento, Italy \\ 2 Multidisciplinary Department of Medico-Surgical and Dentistry \\ Specialties, “Luigi Vanvitelli” University, Naples, Italy \\ ${ }^{3}$ Department of Orthopaedic and Trauma Surgery, AOU Careggi, \\ Florence, Italy \\ ${ }^{4}$ Concordia Hospital for Special Surgery, Rome, Italy \\ Joints 2019;7:107-114.
}

\author{
Giovanni Di Giacomo ${ }^{4}$ Giorgio Benigni ${ }^{1}$
}

\begin{abstract}
Address for correspondence Erica Bulgheroni, MD, Department of Orthopaedic and Trauma Surgery, Santa Chiara Hospital, Largo Medaglie D’Oro g, 38122, Trento, Italy, (e-mail: ericabulgheroni@hotmail.it).
\end{abstract}

\begin{abstract}
Keywords

- patellar dislocation

- medial patellofemoral ligament

- medial patellofemoral reconstruction

- patellar instability

First patellar dislocation is a common injury of the knee, involving often adolescents and the active population. The consequences of the first episode can be various and potentially disabling. Among these, acute patellar dislocation can often result in recurrent patellar instability. Recurrent patellar instability is certainly multifactorial but depends primarily on the injury of the medial patellofemoral ligament (MPFL), the major soft-tissue stabilizer of the patella. Some classifications are extremely useful in establishing the diagnosis and therapy in patellofemoral disease, in particular in terms of instability. Among those, Henri Dejour and WARPS (weak atraumatic risky anatomy pain and subluxation)/STAID (strong traumatic anatomy normal instability and dislocation) classifications are certainly the most frequently used. There is no clear agreement on the management of the first patellar dislocation. A conservative approach seems to be the first choice in most of cases, but the presence of patellar displacement or osteochondral fractures makes surgery mandatory at the beginning. In addition, there is no clear consensus on which surgical strategy should be used to approach first dislocation, in relation to the possible variation in location of the MPFL injury, and to the eventual presence of preexisting predisposing factors for patellar instability. MPFL reconstruction may theoretically be more reliable than repair, while there is no clear evidence available that osseous abnormality should be addressed after the first episode of patellar dislocation. A narrative review was conducted to report the etiology, the diagnosis and all the possible treatment options of the first patellar dislocation. Modern classifications of the patellofemoral instability were also presented.
\end{abstract}

\section{Introduction}

The average incidence of first patellar dislocation is 5.8 cases per 100,000 persons per year, and the rate is higher for adolescents and the active population. ${ }^{1-3}$ It is more common in females, with an incidence of 104 cases per 100,000 persons per year. ${ }^{4}$ First patellar dislocation can lead to disabling pain and decreased activity level, osteochondral fractures, articular cartilage injury, and consequent patellofemoral arthritis, recurrent patellar instability. ${ }^{1,2,5-9}$ The percentage of recurrent patellar dislocation after the first episode varies from 15 to $80 \%,{ }^{1,10,11}$ whereas after the second episode of dislocation, the possibility of recurrence grows to $>50 \%{ }^{1,5}$ Recurrent instability is certainly multifactorial but depends primarily on the injury of the medial patellofemoral ligament (MPFL). It is universally recognized that the MPFL represents the main received

June 13, 2018

accepted after revision

November 4, 2019

published online

December 31, 2019
DOI https://doi.org/

$10.1055 / \mathrm{s}-0039-3401817$.

ISSN 2282-4324.

\footnotetext{
(C) 2019. The Author(s).

This is an open access article published by Thieme under the terms of the Creative Commons Attribution-NonDerivative-NonCommercial-License, permitting copying and reproduction so long as the original work is given appropriate credit. Contents may not be used for commercial purposes, or adapted, remixed, transformed or built upon. (https://creativecommons.org/ licenses/by-nc-nd/4.0/) Georg Thieme Verlag KG, Rüdigerstraße 14, 70469 Stuttgart, Germany
} 
restraint against lateral patellar displacement, ${ }^{12}$ providing approximately 50 to $60 \%$ of the restraining force. ${ }^{13}$ The MPFL insertion at the patella is up to $30 \mathrm{~mm}$ wide and is located along the upper half of the patella. Proximal fibers are mainly attached to the vastus intermedius, without tight adhesion to the vastus medialis. Distal fibers are interdigitated with the deep layer of the medial retinaculum. The femoral insertion of the MPFL is approximately $10 \mathrm{~mm}$ distal to the apex of the adductor tubercle and $16 \mathrm{~mm}$ proximal to the medial epicondyle. ${ }^{14}$ The proximal patellar overlap region makes the MPFL also a dynamic stabilizer, ${ }^{15,16}$ acting principally from 0 to 30 degrees of knee flection. ${ }^{17-19}$ MPFL injury occurs frequently (up to $100 \%$ ) just after the first patellar dislocation. ${ }^{20}$ Anyway, a variable amount of other anatomic abnormalities may contribute to the development of recurrent instability, such as trochlear dysplasia, patella alta or lateral patellar tilt, valgus limb alignment, torsional deformities of the femoral and/or tibia, absence of integrity and/or proper functioning of static, and dynamic soft-tissue constraints. ${ }^{13,21}$ This is certainly a reason of the difficulty in approaching the first patellar dislocation. Additionally, the patient population is very heterogeneous, whereas the surgical and conservative managing techniques are numerous and often challenging, and there are no long-term studies on this topic. ${ }^{1,22}$ Most of the studies seem to indicate a conservative approach as the first choice for most cases, but the presence of patellar displacement or osteochondral fractures can make surgery necessary just after the first dislocation. ${ }^{13}$ In addition, there is no clear consensus on which surgical strategy should be used, even though MPFL reconstruction could theoretically be more reliable than repair. Finally, there is no clear evidence available that osseous abnormality should be managed after the first episode of patellar dislocation.

A narrative review was therefore conducted to report the etiology, the diagnosis and the management of the first patellar dislocation. Conservative versus surgical approach was discussed, and different surgical approaches were presented.

\section{Classification of the Patellofemoral Instability}

In 1987, Dejour et $\mathrm{al}^{23,24}$ described four major instability anatomical factors with a statistical correlation and four minor instability anatomical factors without a statistical correlation causing patellofemoral instability. Major instability anatomical factors include trochlea dysplasia, patella alta, patellar tilt, and pathological tibial tuberosity and trochlear groove (TT-TG) distance. Minor instability anatomical factors include torsional defects of the femur, torsional defects of the tibia, curved knee, and valgus knee. Based on this anatomical factors Dejour et al classified the patellofemoral disease in three patterns objective patellar instability (OPI), potential patellar instability (PPI), and painful patella syndrome (PPS). ${ }^{23,24}$ OPI is represented by the presence of at least one episode of patellar dislocation or subluxation and at least one of the four main factors of instability. PPI is represented by the presence by no episode of patellar dislocation or subluxation but at least one of the four main factors of instability. In PPS, neither episode of patellar dislocation or subluxation is present nor factors of instability, whereas pain is the main symptom.

Another classification system has recently been introduced by Hiemstra et $\mathrm{al}^{25}$ for patellofemoral instability. Similar to shoulder instability, patients with patellofemoral instability can present traumatic or nontraumatic etiology. ${ }^{26}$ Similarly to traumatic anterior shoulder instability/atraumatic multidirectional bilateral rehabilitation inferior capsular shift subset of patients for shoulder pathology, in patellofemoral instability, two main subsets of patients' existence are WARPS and STAID; WARPS is intended how "weak atraumatic risky anatomy pain and subluxation." These patients demonstrate poor femoral quadriceps with poor neuromuscular control, generally atraumatic onset of their instability episodes, many anatomical features possibly responsible for instability, often they complain of pain and subluxations rather than true dislocations.

STAID is intended how "strong traumatic anatomy normal instability and dislocation." Usually these patients tend to have a strong quadriceps with good neuromuscular control, the onset is traumatic and they do not have predispositions factors. They complain of instability with true episodes of dislocation.

\section{Etiology and Diagnosis of the First Patellar Dislocation}

Usually, first patellar dislocation occurs in terminal extension with an axial valgus stress to the knee during rotation, when the femur rotates internally and the tibia externally with the foot fixed on the ground. ${ }^{13,27}$ When the patella dislocates laterally, the medial patellar restraints are injured, particularly the MPFL. The patella may spontaneously relocate back to the femoral trochlear groove; sometimes, a dislocated or a severe lateralized (and tilted) patella may remain. The first patellar dislocation is generally traumatic, although some constitutional anatomic factors may be involved as trochlear dysplasia, patella alta, patellar tilt, TTGG. ${ }^{13,21,23,24}$ All these factors, associated to the MPFL injury, following the first patella dislocation, are well-recognized risk factors for recurrent patellar instability. Rarely, constitutional ligamentous laxity or insufficiency of the medial soft-tissue restraints can be present.

Patellar dislocation is usually diagnosed on the basis of the clinical and anamnestic findings, whereas the patient may describe "kneecap was out of place." Clinical findings include tenderness and pain at the site of the medial restraints. A medial hematoma and a lateralized patella may be observed. Apprehension test is clearly positive. Sometimes, the knee may be swollen due to hemarthrosis, although this sign is not very frequent since the injured medial restraints and MPFL are principally extrasynovial. As mentioned, usually the patella is spontaneously reduced, and remains quite rarely still dislocated when the patient arrives at the hospital.

Standard knee radiographs and magnetic resonance imaging(MRI) are mandatory. Standard plain radiographs, including Merchant's view, are always needed to evaluate patellar position and assess osteochondral fractures or detachments. The patella may be lateralized if compared with the contralateral side and fragmentation of the medial patella, defects of the 
patellar articular surface, or loose osteochondral fragments may be observed. ${ }^{13}$ MRI is useful to assess more precisely the cartilage, to show the MPFL lesion and to identify its location. ${ }^{28-30}$ MPFL lesions are divided in four types as follows: (1) at patellar insertion, (2) midsubstance, (3) femoral origin and (4) a combination. ${ }^{28}$ Both radiographs and MRI are finally useful to evaluate the presence of constitutional bone deformities of the patellofemoral joint that may contribute to recurrent patellar instability. There is certainly a role for computed tomography (CT) scan. ${ }^{21}$ Because of the relatively high incidence of osteochondral fractures after the first patellar dislocation, CT scan is certainly a useful and largely available tool in the emergency room setting to detect osteochondral defects or a patellar bony avulsions when suspected on plain radiographs. ${ }^{30}$ Obviously, CT scan remains the gold standard to detect and measure the TT-TG distance, patellar tilt, and trochlear dysplasia and, in evaluating femoral torsional deformities ${ }^{18}$ in patients with patellofemoral instability.

\section{Conservative Treatment}

High-level evidence supports the effectiveness of the conservative treatment after first patellar dislocation, ${ }^{11}$ especially for the MPFL-vastus medialis obliquus overlap-region lesions, since this region is wide at the attachment to the patella and has a great possibility to spontaneously heal. ${ }^{15}$ However, no randomized controlled clinical trial has been published about different nonoperative approaches. ${ }^{31}$ Some authors suggest cold packs during the first 24 hours, immobilization, and, when necessary, aspiration of the knee effusion, to reduce pain and to allow an easier clinical examination and instrumental diagnosis. ${ }^{13,32}$ Anyway, there is no clear evidence that the knee should be immobilized after patellar dislocation. ${ }^{13}$ Moreover, there is not a consensus on the exact period of knee immobilization, ranging generally from 0 to 6 weeks, ${ }^{8,31-35}$ and the degree of motion allowed by bracing. Some authors suggest an extension position, ${ }^{11}$ others start from 30 degrees of flexion and increase to 90 degrees of flexion after some weeks $^{36}$; consequently, also the type of knee brace proposed may be different in different studies. ${ }^{11,36}$ Maenpaa et $\mathrm{al}^{37}$ failed to find differences between immediate mobilization and flexion restriction in terms of functional results in 72 patients treated conservatively after a first patellar dislocation.

Most of authors agree about the necessity of kinesiotherapy to recover full range of motion (ROM) and quadriceps muscle strengthening to restore the dynamic component of the patellar soft-tissue stabilizers. ${ }^{11,36,38,39}$ No author provides a precise description of the specific exercises or exercise parameters. ${ }^{11,32,36}$ The most common strategy includes isometric and isotonic quadriceps strengthening exercises, such as straight leg raises, squats, and lunges. ${ }^{31}$ Apostolovic et $\mathrm{al}^{32}$ suggests principally the utilization of isometric quadriceps exercises. Anyway, there is little evidence to support a specific exercise program for preventing future dislocations. ${ }^{40}$ The amount of exercise should rise with time as tolerated by pain, and at 4 to 6 weeks, generally, the patient can walk with a complete ROM. Full work and/or sport activity is generally allowed after 3 months. ${ }^{13}$
The clinical and functional results and the redislocation rate after conservative treatment of the first patellar dislocations are variable. In a recent systematic review on 2,086 patients treated for a first patellar dislocation, Longo et $\mathrm{al}^{40}$ reported an average Kujula's score of 75.6 points at short-medium-term follow-up ( $<5$ years) and 87.5 points at long-term follow-up ( $>5$ years) in the patients managed conservatively; the rate of dislocation recurrence was $36.4 \%$, while no other complication was reported. Ji et $\mathrm{al}^{41}$ reported the results of the conservative treatment in 30 patients, the mean Kujula score was 93.6 points at the latest follow-up, while the mean patellar tilt was 6.8 degrees, and lateral shift ratio was 9.2. Regalado et $\mathrm{al}^{36}$ analyzing the results of 20 patients treated conservatively after a first patellar dislocation, found a $27 \%$ of poor knee function and a $27 \%$ of unsatisfied patients, with a redislocation rate of 35 and $73 \%$ at 3 and 6 years, respectively.

\section{Surgical Treatment}

The circumstances that could immediately lead to surgery after a first patellar dislocation include the presence of osteochondral fragments, important cartilage damage, disruption of the medial stabilizers with subluxation, or complete displacement of the patella. ${ }^{21}$ It has been proposed that a MPFL lesion at its femoral origin would need an immediate surgical approach ${ }^{15}$; in this area, the ligament is thin and its retraction after rupture can change femoral attachment point leading to low-medial restrain. There is still controversy about the choice of repairing rather than reconstructing the torn MPFL after the first patellar dislocation. A careful clinical, instrumental (radiographs plus MRI) and arthroscopic examination is needed to decide the appropriate treatment (MPFL repair vs. reconstruction); in fact, the main cause of unsatisfying results is that MPFL injuries can have different patterns and locations. ${ }^{27}$ The MPFL patellar attachment can be surgically reinserted with a good functional outcome restoring the anatomical insertion. ${ }^{27}$ On the other hand, for the MPFL lesion at its femoral origin the repair could be more challenging due to the need to optimize the insertion point on the femur, as small changes in positioning, can cause increased stress on the repaired tissue and lead to failure. ${ }^{1}$ Midsubstance MPFL injury is difficult to repair adequately, so that is generally not recommended. ${ }^{27,42}$

\section{MPFL Repair}

Some authors support the rule of the MPFL repair after first patellar dislocation. ${ }^{34,42,43}$ MPFL repair can be performed only if the location of the lesion is known ${ }^{27,44}$ and, generally, within three weeks from trauma. ${ }^{1,35}$ As mentioned above, MPFL injury at patellar origin could be repaired. Patellar MPFL lesion can be classified as ligamentous or bony avulsion or associated with an osteochondral fragment with articular cartilage involvement. ${ }^{13,44}$ Obviously, the last two types always need a surgical approach. ${ }^{45,46}$ Specifically, fractures located in a high-pressure area should be repaired if possible. Differently, if the fracture is located in a low-pressure area, the fragment may be removed. ${ }^{44}$ The authors that support this technique assure that MPFL repair at its patellar origin allows to restore the anatomical position of the ligament, 
without complications, achieving a good tension of the ligament itself. ${ }^{2}$

Numerous open and arthroscopic MPFL repair techniques have been described. ${ }^{47-51}$ Arthroscopic MPFL repair may be technically demanding and can fully visualize only the patella-based injuries; additionally, the arthroscopic approach leaves suture material into the joint, with possible irritation. ${ }^{52}$ In the literature, reports show a redislocation rate after arthroscopic medial retinacular repair/plication based on similar techniques ranging from 0 to $19 \% .^{51-53}$ Arendt et $\mathrm{al}^{35}$ and Matic et $\mathrm{al}^{54}$ found that almost half of the patients who experience recurrent dislocation after MPFL repair are an average of 4 years younger (at the time of surgery) than those who do not undergo a redislocation, probably in relation to the higher activity level in younger patients. Several studies in literature report good clinical results and low redislocation rates after MPFL repair. Among the studies relative to the MPFL reinsertion to the patellar origin, Small et $\mathrm{al}^{55}$ reported a $92 \%$ of good or excellent subjective results in 25 of the 27 knees, while Halbrecht ${ }^{52}$ reported a 93\% of good subjective and objective results in 26 patients with no case of redislocation. Yamamoto ${ }^{56}$ reported only one case of redislocation on 30 (3.3\%) patients treated with an arthroscopic MPFL repair, while Schöttle et $\mathrm{al}^{57}$ reported four cases of redislocation on 91 (4.4\%) patients treated with an arthroscopic MPFL repair. With the same technique, Haspl et $\mathrm{al}^{53}$ reported no case $(0 \%)$ of redislocation.

About the MPFL repair to the femoral origin, Sallay et $\mathrm{al}^{58}$ reported no case of redislocation at an average of 34 months and $58 \%$ of the results were considered good or excellent; $58 \%$ of the patients returned to their previous sportive activity. Ahmad et al $^{59}$ reported no redislocation at a mean of 3 years of follow-up, with an average Kujala's score of 91.9 points and a subjective satisfaction of $96 \%$ of the patients; $86 \%$ of the patients returned to their preinjury athletic activity level.

Christiansen et $\mathrm{al}^{48}$ found a redislocation rate of 17 and $20 \%$ in operative and conservative group of patients, respectively, a Kujala score of 85 and 78 points, a patella stability score significantly higher in the operative group, and no difference in Knee Injury and Osteoarthritis Scores.

Finally, some Authors reported that both MPFL repair is effective in providing the preinjury level of activity, although failure rate, defined as recurrent patellar instability, is higher when compared with MPFL reconstruction. ${ }^{54}$

\section{MPFL Reconstruction}

MPFL reconstruction is used 1.5 times more often than repair or medial retinacular/plication and seems to provide more consistent outcomes after first patellar dislocation, both in terms of clinical outcomes and rate of redislocation. ${ }^{44,60,61}$ Most of Authors prefer to reconstruct the MPFL due to the better quality of collagen-containing graft rather than the stretched or compromised tissues. ${ }^{22}$ Numerous techniques for MPFL reconstruction have been described, and they all aim to supply tendon tissue from the medial aspect of the patella to the femoral insertion site of the native MPFL. ${ }^{20}$
Panagopoulos et $\mathrm{al}^{62}$ and Xie et $\mathrm{al}^{63}$ reported a great and significant increase in Tegner's score with MPFL reconstruction using semitendinosus tendon graft on 25 and 85 knees, respectively; additionally, Panagopoulos reported no case of redislocation, while Xie had a redislocation rate of $1.1 \%$. In two different systematic reviews considering different surgical approaches, Matic et $\mathrm{al}^{54}$ and Kuroda et $\mathrm{al}^{64}$ reported more reliable outcomes with reconstruction than with repair in multifocal and midsubstance MPFL injury and in high-level athletes. In particular, Matic et $\mathrm{al}^{54}$ found the redislocation rate four times higher with MPFL repair and 2.5 times higher with medial retinacular repair/plication when compared with MPFL reconstruction (6.6\%). Anyway, the authors reported a complete return to the preinjury activities for athletes independently from the type of surgery at approximately 5.1 months. In another systematic review including 25 papers about the different surgical techniques used for managing the first patellar dislocation including additional bone procedures, Shah et $\mathrm{al}^{65}$ reported a lower redislocation rate $(3.7 \%)$ in patients treated with MPFL reconstruction (alone).

\section{Surgical versus Conservative Approach}

When compared with the conservative treatment, the overall results of the surgical approach after first patellar dislocation are not clear, whereas the redislocation rate varies from 13 to $52 \%$ and good subjective scores vary from 47 to $85 \%{ }^{15,66}$ The possibility of failure seems to be related with young age and high-activity level. ${ }^{31,35}$ Longo et $\mathrm{al}^{40}$ suggested that surgical treatment has a lower rate of redislocation and provides better short-medium-term clinical outcomes when compared with conservative treatment, whereas in long-term follow-up, the results are similar. Smith et $\mathrm{al}^{67}$ found that surgical treatment of first dislocation is associated to a lower degree of patellar redislocation but to a greater risk of successive patellofemoral osteoarthritis. This could be related to some techniques that could change the kinematic of the patellofemoral joint, therefore favoring a successive joint degeneration. Christiansen et $\mathrm{al}^{48}$ reported a redislocation rate of 16.7 and $20 \%$ in the surgical and in the conservative group, respectively, although in absence of better subjective functional outcomes in the surgical group. Nikku et $\mathrm{al}^{42}$ reported a redislocation rate at 7 years of 31 and 39\% for the operative and nonoperative group, respectively. At a 14 -year follow-up, Palmu et al ${ }^{11}$ reported a similar redislocation rates (71 vs. $67 \%$ ) in the conservative group and surgical group, respectively. However, it has to be considered how the above-mentioned studies included different surgical techniques and different conservative strategies. - Table 1 include all the details, type of treatment, results, and percentage of patellar redislocation of the more significant mentioned clinical studies.

\section{Treatment of Associated Bone Deformities}

It is well recognized that when performing a surgical intervention for recurrent patellar instability, one should take into account the soft-tissue stabilizers disrupted during dislocation, as well as anatomical abnormalities that may 


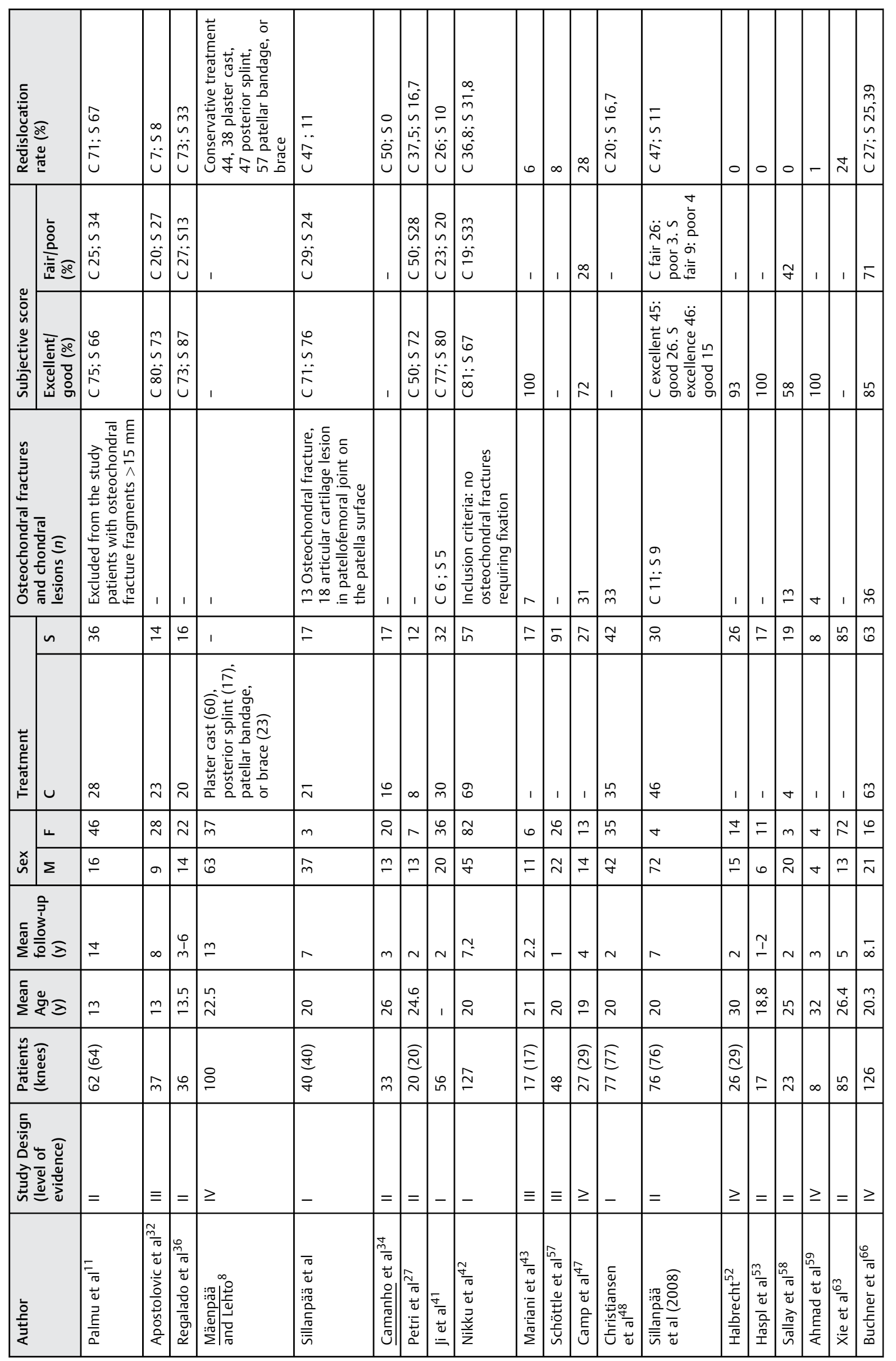

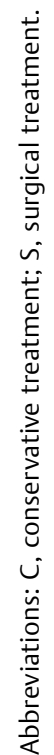


predispose to chronic dislocation. ${ }^{13}$ Differently, bone procedures are generally not considered a first-line treatment after the first patella dislocation; there is no evidence that osseous abnormalities should be addressed in addition to MPFL repair/reconstruction after the first episode of dislocation. ${ }^{68}$ In particular, in adolescents, a trochlear dysplasia or a malalignment will be treated in a second time. ${ }^{68}$ Sillanpää et $\mathrm{al}^{4}$ suggested that osseous surgery is not necessary after primary dislocation, although bone corrections may become needed in cases of severe abnormalities. On the other hand, it has been reported how clinical and functional outcomes following the first patella dislocation could vary in relation to the presence of risk factors for instability, such as trochlear dysplasia, patella malalignment, and axial and torsional lower limb alignment abnormalities. ${ }^{24,69,70}$ It has been reported that the more dysplastic is the trochlear shape, the more an MPFL injury is problematic in terms of (recurrent) patellar instability. ${ }^{27}$ Therefore, in rare cases of severe associated anatomic patellofemoral deformities, procedure like tibial tuberosity transpositions and/or trochleoplasty can be taken into consideration and associated to MPFL repair/reconstruction. ${ }^{27}$

\section{Conclusion}

There is not a clear consensus on the management of the first patellar dislocation. Conservative approach has been advocated in most of cases, with good results in terms of functional outcomes and rate of redislocation. Surgery is certainly needed in presence of severe patella displacement or osteochondral fragments and seems to provide lower rates of patellar redislocation when compared with conservative treatment. Repair of the torn MPFL may be preferred in case of (bony) avulsion from the patella; anyway, MPFL reconstruction has been shown to be more reliable than repair. Finally, there is no evidence that bone abnormalities should be corrected after the first episode of patella dislocation, although in rare cases of severe associated anatomic patellofemoral deformities, procedures like tibial tuberosity transpositions and/or trochleoplasty can be taken into consideration and associated to MPFL repair/ reconstruction.

Conflict of Interest

None declared.

\section{References}

1 Fithian DC, Paxton EW, Stone ML, et al. Epidemiology and natural history of acute patellar dislocation. Am J Sports Med 2004;32 (05):1114-1121

2 Hawkins RJ, Bell RH, Anisette G. Acute patellar dislocations. The natural history. Am J Sports Med 1986;14(02):117-120

3 Hsiao M, Owens BD, Burks R, Sturdivant RX, Cameron KL. Incidence of acute traumatic patellar dislocation among active-duty United States military service members. Am J Sports Med 2010;38 (10):1997-2004

4 Sillanpää P, Mattila VM, Iivonen T, Visuri T, Pihlajamäki H. Incidence and risk factors of acute traumatic primary patellar dislocation. Med Sci Sports Exerc 2008;40(04):606-611
5 Arendt EA, Fithian DC, Cohen E. Current concepts of lateral patella dislocation. Clin Sports Med 2002;21(03):499-519

6 Frandsen PA, Kristensen H. Osteochondral fracture associated with dislocation of the patella: another mechanism of injury. J Trauma 1979;19(03):195-197

7 Larsen E, Lauridsen F. Conservative treatment of patellar dislocations. Influence of evident factors on the tendency to redislocation and the therapeutic result. Clin Orthop Relat Res 1982; (171):131-136

8 Mäenpää $\mathrm{H}$, Lehto MU. Patellar dislocation. The long-term results of nonoperative management in 100 patients. Am J Sports Med 1997;25(02):213-217

9 Seeley M, Bowman KF, Walsh C, Sabb BJ, Vanderhave KL. Magnetic resonance imaging of acute patellar dislocation in children: patterns of injury and risk factors for recurrence. J Pediatr Ortop 2012;32(02):145-155

10 Garth WP Jr, DiChristina DG, Holt G. Delayed proximal repair and distal realignment after patellar dislocation. Clin Orthop Relat Res 2000;(377):132-144

11 Palmu S, Kallio PE, Donell ST, Helenius I, Nietosvaara Y. Acute patellar dislocation in children and adolescents: a randomized clinical trial. J Bone Joint Surg Am 2008;90(03):463-470

12 Panni AS, Cerciello S, Vasso M. Patellofemoral instability: surgical treatment of soft tissues. Joints 2013;1(01):34-39

13 Sillanpää PJ, Mäenpää HM. First-time patellar dislocation: surgery or conservative treatment? Sports Med Arthrosc Rev 2012;20 (03):128-135

14 Mochizuki T, Nimura A, Tateishi T, Yamaguchi K, Muneta T, Akita K. Anatomic study of the attachment of the medial patellofemoral ligament and its characteristic relationships to the vastus intermedius. Knee Surg Sports Traumatol Arthrosc 2013;21(02): 305-310

15 Kang HJ, Wang F, Chen BC, Zhang YZ, Ma L. Non-surgical treatment for acute patellar dislocation with special emphasis on the MPFL injury patterns. Knee Surg Sports Traumatol Arthrosc 2013;21 (02):325-331

16 Panni AS, Alam M, Cerciello S, Vasso M, Maffulli N. Medial patellofemoral ligament reconstruction with a divergent patellar transverse 2-tunnel technique. Am J Sports Med 2011;39(12): 2647-2655

17 Conlan T, Garth WP Jr, Lemons JE. Evaluation of the medial softtissue restraints of the extensor mechanism of the knee. J Bone Joint Surg Am 1993;75(05):682-693

18 Desio SM, Burks RT, Bachus KN. Soft tissue restraints to lateral patellar translation in the human knee. Am J Sports Med 1998;26 (01):59-65

19 Hautamaa PV, Fithian DC, Kaufman KR, Daniel DM, Pohlmeyer AM. Medial soft tissue restraints in lateral patellar instability and repair. Clin Orthop Relat Res 1998;(349):174-182

20 Vasso M, Corona K, Toro G, Rossini M, Schiavone Panni A. Anatomic Double-Bundle Medial Patellofemoral Ligament Reconstruction with Autologous Semitendinosus: Aperture Fixation Both at the Femur and the Patella. Joints 2017;5(04):256-260

21 Stefancin JJ, Parker RD. First-time traumatic patellar dislocation: a systematic review. Clin Orthop Relat Res 2007;455(455):93-101

22 Weber AE, Nathani A, Dines JS, et al. An Algorithmic Approach to the Management of Recurrent Lateral Patellar Dislocation. J Bone Joint Surg Am 2016;98(05):417-427

23 Dejour H, Walch G, Neyret P, Adeleine P. [Dysplasia of the femoral trochlea]. Rev Chir Orthop Repar Appar Mot 1990;76(01):45-54

24 Dejour H, Walch G, Nove-Josserand L, Guier C. Factors of patellar instability: an anatomic radiographic study. Knee Surg Sports Traumatol Arthrosc 1994;2(01):19-26

25 Hiemstra LA, Kerslake S, Lafave M, Heard SM, Buchko GM. Introduction of a classification system for patients with patellofemoral instability (WARPS and STAID). Knee Surg Sports Traumatol Arthrosc 2014;22(11):2776-2782 
26 Andrish J. Surgical options for patellar stabilization in the skeletally immature patient. Sports Med Arthrosc Rev 2007;15(02):82-88

27 Petri M, von Falck C, Broese M, et al. Influence of rupture patterns of the medial patellofemoral ligament (MPFL) on the outcome after operative treatment of traumatic patellar dislocation. Knee Surg Sports Traumatol Arthrosc 2013;21(03):683-689

28 Balcarek P, Walde TA, Frosch S, Schüttrumpf JP, Wachowski MM, Stürmer KM. MRI but not arthroscopy accurately diagnoses femoral MPFL injury in first-time patellar dislocations. Knee Surg Sports Traumatol Arthrosc 2012;20(08):1575-1580

29 Elias DA, White LM, Fithian DC. Acute lateral patellar dislocation at MR imaging: injury patterns of medial patellar soft-tissue restraints and osteochondral injuries of the inferomedial patella. Radiology 2002;225(03):736-743

30 Smith TO, Drew BT, Toms AP, Donell ST, Hing CB. Accuracy of magnetic resonance imaging, magnetic resonance arthrography and computed tomography for the detection of chondral lesions of the knee. Knee Surg Sports Traumatol Arthrosc 2012;20(12): 2367-2379

31 Smith TO, Davies L, Chester R, Clark A, Donell ST. Clinical outcomes of rehabilitation for patients following lateral patellar dislocation: a systematic review. Physiotherapy 2010;96(04):269-281

32 Apostolovic M, Vukomanovic B, Slavkovic N, et al. Acute patellar dislocation in adolescents: operative versus nonoperative treatment. Int Orthop 2011;35(10):1483-1487

33 Sillanpää PJ, Mattila VM, Mäenpää H, Kiuru M, Visuri T, Pihlajamäki H. Treatment with and without initial stabilizing surgery for primary traumatic patellar dislocation. A prospective randomized study. J Bone Joint Surg Am 2009;91(02):263-273

34 Camanho GL, Viegas AdeC, Bitar AC, Demange MK, Hernandez AJ. Conservative versus surgical treatment for repair of the medial patellofemoral ligament in acute dislocations of the patella. Arthroscopy 2009;25(06):620-625

35 Arendt EA, Moeller A, Agel J. Clinical outcomes of medial patellofemoral ligament repair in recurrent (chronic) lateral patella dislocations. Knee Surg Sports Traumatol Arthrosc 2011;19(11):1909-1914

36 Regalado G, Lintula H, Kokki H, Kröger H, Väätäinen U, Eskelinen M. Six-year outcome after non-surgical versus surgical treatment of acute primary patellar dislocation in adolescents: a prospective randomized trial. Knee Surg Sports Traumatol Arthrosc 2016;24 (01):6-11

37 Maenpaa H, Sillanpaa PJ, Paakkala A. a Prospective, randomized trial following conservative treatment in acute primary patellar dislocation with special reference to patellar braces. Knee Surg Sports Traumatol Arthrosc 2010;18(Suppl 1):119

38 Greiwe RM, Saifi C, Ahmad CS, et al. Anatomy and biomechanics of patellar instability. Oper Tech Sports Med 2010;18:62-67

39 McConnell J. Rehabilitation and nonoperative treatment of patellar instability. Sports Med Arthrosc Rev 2007;15(02):95-104

40 Longo UG, Ciuffreda M, Locher J, Berton A, Salvatore G, Denaro V. Treatment of primary acute patellar dislocation: systematic review and quantitative synthesis of the literature. Clin J Sport Med 2017; 27(06):511-523

41 Ji G, Wang S, Wang X, Liu J, Niu J, Wang F. Surgical versus nonsurgical treatments of acute primary patellar dislocation with special emphasis on the MPFL injury patterns. J Knee Surg 2017;30(04):378-384

42 Nikku R, Nietosvaara Y, Aalto K, Kallio PE. Operative treatment of primary patellar dislocation does not improve medium-term outcome: A 7-year follow-up report and risk analysis of 127 randomized patients. Acta Orthop 2005;76(05):699-704

43 Mariani PP, Liguori L, Cerullo G, Iannella G, Floris L. Arthroscopic patellar reinsertion of the MPFL in acute patellar dislocations. Knee Surg Sports Traumatol Arthrosc 2011;19(04):628-633

44 Sillanpää PJ, Peltola E, Mattila VM, Kiuru M, Visuri T, Pihlajamäki H. Femoral avulsion of the medial patellofemoral ligament after primary traumatic patellar dislocation predicts subsequent insta- bility in men: a mean 7-year nonoperative follow-up study. Am J Sports Med 2009;37(08):1513-1521

45 Slabaugh MA, Hess DJ, Bajaj S, et al. Management of chondral injuries associated with patellar instability. Oper Tech Sports Med 2010;18:115-122

46 Mehta VM, Inoue M, Nomura E, Fithian DC. An algorithm guiding the evaluation and treatment of acute primary patellar dislocations. Sports Med Arthrosc Rev 2007;15(02):78-81

47 Camp CL, Krych AJ, Dahm DL, Levy BA, Stuart MJ. Medial patellofemoral ligament repair for recurrent patellar dislocation. Am J Sports Med 2010;38(11):2248-2254

48 Christiansen SE, Jakobsen BW, Lund B, Lind M. Isolated repair of the medial patellofemoral ligament in primary dislocation of the patella: a prospective randomized study. Arthroscopy 2008;24 (08):881-887

49 Dodson CC, Shindle MK, Dines JS, Altchek DW. Arthroscopic suture anchor repair for lateral patellar instability. Knee Surg Sports Traumatol Arthrosc 2010;18(02):143-146

50 Fukushima K, Horaguchi T, Okano T, Yoshimatsu T, Saito A, Ryu J. Patellar dislocation: arthroscopic patellar stabilization with anchor sutures. Arthroscopy 2004;20(07):761-764

51 Sillanpää PJ, Mäenpää HM, Mattila VM, Visuri T, Pihlajamäki H. Arthroscopic surgery for primary traumatic patellar dislocation: a prospective, nonrandomized study comparing patients treated with and without acute arthroscopic stabilization with a median 7-year follow-up. Am J Sports Med 2008;36(12):2301-2309

52 Halbrecht JL. Arthroscopic patella realignment: an all-inside technique. Arthroscopy 2001;17(09):940-945

53 Haspl M, cicak N, Klobucar H, Pecina M. Fully arthroscopic stabilization of the patella. Arthroscopy 2002;18(01):E2

54 Matic GT, Magnussen RA, Kolovich GP, Flanigan DC. Return to activity after medial patellofemoral ligament repair or reconstruction. Arthroscopy 2014;30(08):1018-1025

55 Small NC, Glogau AI, Berezin MA. Arthroscopically assisted proximal extensor mechanism realignment of the knee. Arthroscopy 1993;9(01):63-67

56 Yamamoto RK. Arthroscopic repair of the medial retinaculum and capsule in acute patellar dislocations. Arthroscopy 1986;2(02): 125-131

57 Schöttle PB, Scheffler SU, Schwarck A, Weiler A. Arthroscopic medial retinacular repair after patellar dislocation with and without underlying trochlear dysplasia: a preliminary report. Arthroscopy 2006;22(11):1192-1198

58 Sallay PI, Poggi J, Speer KP, Garrett WE. Acute dislocation of the patella. A correlative pathoanatomic study. Am J Sports Med 1996;24(01):52-60

59 Ahmad CS, Stein BE, Matuz D, Henry JH. Immediate surgical repair of the medial patellar stabilizers for acute patellar dislocation. A review of eight cases. Am J Sports Med 2000;28(06):804-810

60 Duchman KR, Bollier MJ. The role of medial patellofemoral ligament repair and imbrication. Am J Orthop 2017;46(02):87-91

61 Smith TO, Walker J, Russell N. Outcomes of medial patellofemoral ligament reconstruction for patellar instability: a systematic review. Knee Surg Sports Traumatol Arthrosc 2007;15(11): 1301-1314

62 Panagopoulos A, van Niekerk L, Triantafillopoulos IK. MPFL reconstruction for recurrent patella dislocation: a new surgical technique and review of the literature. Int J Sports Med 2008;29 (05):359-365

63 Xie G, Zhao J, Huangfu X, He Y. Medial patellofemoral ligament reconstruction using semitendinosus tendons: polyester suture augmentation versus nonaugmentation. Am J Sports Med 2012; 40(06):1365-1374

64 Kuroda Y, Matsushita T, Matsumoto T, Kawakami Y, Kurosaka M, Kuroda R. Bilateral medial patellofemoral ligament reconstruction in high-level athletes. Knee Surg Sports Traumatol Arthrosc 2014;22(10):2465-2469 
114 First Patellar Dislocation Bulgheroni et al.

65 Shah JN, Howard JS, Flanigan DC, Brophy RH, Carey JL, Lattermann C. A systematic review of complications and failures associated with medial patellofemoral ligament reconstruction for recurrent patellar dislocation. Am J Sports Med 2012;40(08):1916-1923

66 Buchner M, Baudendistel B, Sabo D, Schmitt H. Acute traumatic primary patellar dislocation: long-term results comparing conservative and surgical treatment. Clin J Sport Med 2005;15(02):62-66

67 Smith TO, Song F, Donell ST, Hing CB. Operative versus nonoperative management of patellar dislocation. A meta-analysis. Knee Surg Sports Traumatol Arthrosc 2011;19(06):988-998
68 Panni AS, Vasso M, Cerciello S. Acute patellar dislocation. What to do? Knee Surg Sports Traumatol Arthrosc 2013;21(02): 275-278

69 Mäenpää $H$, Lehto MU. Patellar dislocation has predisposing factors. A roentgenographic study on lateral and tangential views in patients and healthy controls. Knee Surg Sports Traumatol Arthrosc 1996;4(04):212-216

70 Teitge RA. Patellofemoral syndrome a paradigm for current surgical strategies. Orthop Clin North Am 2008;39(03): 287-311 